\title{
Combined Analysis of Serum Alpha-Fetoprotein and MAGE-A3-Specific Cytotoxic T Lymphocytes in Peripheral Blood for Diagnosis of Hepatocellular Carcinoma
}

\author{
Zhuqingqing Cui, ${ }^{1}$ Xin Yu, ${ }^{1}$ Limin Guo, ${ }^{2}$ Yuhua Wei, ${ }^{1}$ Shengmin Zheng, ${ }^{1}$ Wenxia Li, \\ Pengcheng Chen, ${ }^{3}$ Jiye Zhu, ${ }^{1}$ and Jirun Peng ${ }^{1}$ \\ ${ }^{1}$ Department of Surgery, Beijing University People's Hospital, No. 11, South Xizhimen Street, Xicheng, Beijing 100044, China \\ 2 Department of Surgery, Beijing University Ditan Hospital, Beijing 100015, China \\ ${ }^{3}$ Department of Surgery, The First Affiliated Hospital of Tsinghua University, Beijing 100016, China
}

Correspondence should be addressed to Jiye Zhu; zhujy001@126.com and Jirun Peng; pengjrpku@gmail.com

Received 20 June 2013; Revised 14 August 2013; Accepted 13 September 2013

Academic Editor: Timothy M. Block

Copyright ( 2013 Zhuqingqing Cui et al. This is an open access article distributed under the Creative Commons Attribution License, which permits unrestricted use, distribution, and reproduction in any medium, provided the original work is properly cited.

\begin{abstract}
We investigated the feasibility of the combined detection of HLA-A2/MAGE-A3 epitope-specific cytotoxic T lymphocytes (CTLs) and serum alpha-fetoprotein (AFP) for specific diagnosis of hepatocellular carcinoma (HCC). We detected the frequency of MAGEA3 epitopes (p112-120, KVAELVHFL) in spontaneous CTLs in the peripheral blood of HCC patients, liver cirrhosis patients, and healthy subjects with HLA-A2/polypeptide complex (pentamer) detection technology. Eighty-five HCC cases, 38 liver cirrhosis cases, and 50 healthy cases who were HLA-A2-positive were selected from 175 HCC patients, 80 patients with liver cirrhosis, and 105 healthy volunteers, respectively. The frequency of HLA-A2-specific MAGE-A $3^{+}$CTLs in the HCC group was significantly higher than that in the other groups. Combined detection of MAGE-A $3^{+}$CTL frequency and serum AFP value had a higher specificity than either of the two indicators alone. The pentamer technique is helpful in distinguishing benign lesions and malignant lesions in the liver. Combined with serum AFP, it can improve the diagnosis performance for HCC, especially for AFP-negative cancer.
\end{abstract}

\section{Introduction}

Hepatocellular carcinoma (HCC) ranks the fifth in incidence globally in men and the ninth in women according to the 2012 report by the American Cancer Society [1]. It has become the fastest growing cause of cancer-related deaths in the United States and is the second leading cause of death in China. The currently recommended treatments include surgical resection, radiofrequency ablation, and liver transplantation. However, in the most advanced stage cases, liver reserve deficiency or the combinations of other liver diseases and complications render these patients unsuitable for surgery [2]. Even after surgical excision, the postoperative recurrence rate within 5 years is more than $50 \%$, and the 5year survival rate is less than $20 \%$. The 5 -year survival rate of patients undergoing liver transplantation has increased in recent years, but the overall prognosis remains unfavorable
[3], indicating that the current treatment options are unsatisfactory. Poor survival of HCC patients is strongly associated with tumor size $[4,5]$, tumor stage [6], vascular invasion [7], and micrometastasis [8], which are reliable and important indicators of tumor growth and invasion. Vascular involvement by HCC cells, causing hematogenous dissemination, may lead to a high rate of recurrence and metastasis and poor prognosis $[9,10]$.

For earlier therapeutic intervention, better early diagnostic techniques are urgently required. At present, imaging examinations such as B-mode ultrasonography, computed tomography (CT), and magnetic resonance imaging (MRI) and serum alpha-fetoprotein (AFP) levels are mainly used to diagnose HCC. Serum AFP is effective and simple for screening HCC. However, accurate diagnosis is difficult in approximately one third of HCC patients with low AFP levels if the imaging findings are atypical [11]. In recent years, great 
progress has been made in screening tumor-specific antigens, which has led to the identification of a variety of candidate proteins and genes. Simpson et al. first reported cancer-testis antigens in melanoma patients [12], which belong to the largest group of identified specific tumor antigens. Cancertestis antigens, including the melanoma antigen (MAGE) family, SSX family, LAGE, GAGE, CTp11, and NY-ESO-1, are generally not expressed in normal tissues except in the testis but are expressed to some degree in HCC, malignant melanoma, lung cancer, sarcoma, and bladder cancer, among other tumor types. These antigens were found to be the most promising class of antigens for immune therapy. Since 1998, our group has studied cancer-testis antigens and HCC, and we have found that MAGE-1, MAGE-3, MAGE-4, MAGE-10, MAGE-B1, MAGE-B2, MAGE-C1, MAGE-C2, NY-ESO-1 or other CT antigens were highly expressed in the tumor tissue of Chinese HCC patients [13-15]. MAGE-3 is not only highly expressed in HCC but also has strong immunogenicity with multiple HLA-restricted epitopes, as identified with tetramer technology. For example, MAGE-3 can be detected in the peripheral blood of patients with gastrointestinal cancer, melanoma, or lung cancer as a specific cellular immune response $[12,16-19]$. Thus, we postulated that pentamer technology could be applied as a diagnostic method for HCC alongside routine examination. MAGE-A3-specific cytotoxic T lymphocyte (CTL) antigens were detectable in the peripheral blood of HCC patients. Combined with serum AFP, we hypothesized that this parameter may provide a more reliable diagnosis for AFP-negative HCC patients.

In this study, we performed pentamer staining and flow cytometry to detect the frequency of HLA-A2/MAGE-A3112120 epitope-specific CTLs in the peripheral blood of HLA-A2positive HCC patients, liver cirrhosis patients, and healthy volunteers. The frequency of CTLs in patients with liver cirrhosis was significantly lower than that of HCC patients $(P<0.05)$, while healthy controls had few CD8/MAGE-A3 double-positive cells. Therefore, this approach may help to distinguish between benign and malignant liver disease and could be combined with serum AFP measurement to form a new diagnostic method for HCC.

\section{Subjects and Methods}

2.1. Subjects. We retrospectively analyzed the clinicopathologic data of $175 \mathrm{HCC}$ patients who underwent surgery at our hospital between June 2007 and December 2012. They included 117 males and 58 females with a mean age of 53.3 \pm 6.8 (range 28 to 84 ) years. Sixty patients were infected with hepatitis $\mathrm{B}$ virus (HBV) and two patients were infected with hepatitis $\mathrm{C}$ virus (HCV). Additionally, 80 cancer-free liver cirrhosis patients were included as controls. HCC tissue and adjacent normal tissue specimens were excised during surgery and were snap frozen in liquid nitrogen and were subsequently classified by pathological examination. Diagnosis of HCC was based on preoperative investigations, which included serum AFP concentration, liver ultrasonography, CT, and hepatic angiography in selected cases. The clinicopathological data of HCC patients are summarized in
TABLE 1: Demographic features and clinicopathologic characteristics of 85 HLA-A2 positive hepatocellular cancer patients and their correlation with the frequency of MAGE-A $3^{+}$CTL.

\begin{tabular}{|c|c|c|c|}
\hline \multirow{2}{*}{ Variables } & \multicolumn{3}{|c|}{ Frequency of MAGE-A3 ${ }^{+}$CTL } \\
\hline & $\geq 0.67 \%$ & $<0.67 \%$ & $P^{*}$ \\
\hline \multicolumn{4}{|l|}{ Gender } \\
\hline Male & 44 & 26 & \multirow{2}{*}{0.781} \\
\hline Female & 10 & 5 & \\
\hline \multicolumn{4}{|l|}{ Age (years) } \\
\hline $66-85$ & 14 & 8 & \multirow{3}{*}{0.413} \\
\hline $46-65$ & 29 & 20 & \\
\hline $28-45$ & 11 & 3 & \\
\hline \multicolumn{4}{|l|}{$\operatorname{AFP}(\mathrm{ng} / \mathrm{mL})$} \\
\hline$<20$ & 20 & 18 & \multirow{3}{*}{0.268} \\
\hline $20-200$ & 11 & 4 & \\
\hline$>200$ & 19 & 9 & \\
\hline \multicolumn{4}{|l|}{ Tumor size } \\
\hline$\geq 5 \mathrm{~cm}$ & 23 & 20 & \multirow{2}{*}{0.057} \\
\hline$<5 \mathrm{~cm}$ & 24 & 8 & \\
\hline \multicolumn{4}{|l|}{ Tumor number } \\
\hline Single & 45 & 28 & \multirow{2}{*}{0.373} \\
\hline Multiple & 9 & 3 & \\
\hline \multicolumn{4}{|l|}{ HBsAg } \\
\hline Positive & 36 & 24 & \multirow{2}{*}{0.295} \\
\hline Negative & 18 & 7 & \\
\hline \multicolumn{4}{|l|}{ Child-Pugh class } \\
\hline A & 40 & 26 & \multirow{3}{*}{0.443} \\
\hline B & 13 & 4 & \\
\hline $\mathrm{C}$ & 1 & 1 & \\
\hline \multicolumn{4}{|l|}{ TNM stage } \\
\hline I/II & 43 & 21 & \multirow{2}{*}{0.221} \\
\hline III/IV & 11 & 10 & \\
\hline \multicolumn{4}{|c|}{ Histological differentiation } \\
\hline Well/moderately & 40 & 26 & \multirow{2}{*}{0.094} \\
\hline Low & 14 & 3 & \\
\hline
\end{tabular}

${ }^{*}$ Chi-square test and Fisher's exact test.

Table 1. Patients received no intervention before surgery. Liver function was assessed using Child-Pugh classification. Histopathological examination of the tissue specimens was conducted by two pathologists to confirm the diagnosis of HCC. Tumor stages were based on the American Joint Committee on Cancer (AJCC) staging system (2010). Diagnosis of cirrhosis was based on liver histology as well as laboratory and imaging evidence of hepatic decompensation or portal hypertension. Furthermore, 105 healthy volunteers were included in the study, all of whom were negative for the markers of hepatitis viruses $\mathrm{A}, \mathrm{B}, \mathrm{C}, \mathrm{D}$, and $\mathrm{E}$ and HIV antibodies, and had no liver, gallbladder, or kidney disease.

The study protocol was approved by the local institutional review boards at the authors' affiliated institutions and patient consent was not required because of the retrospective nature of this study. 
2.2. HLA Phenotype Analysis. Two $\mathrm{mL}$ peripheral venous blood was collected from each subject and HLA phenotyping was performed using HLA-A2 fluorescein isothiocyanate (FITC) conjugated mouse anti-human monoclonal antibody (BB7.2 ATCC; Becton Dickinson, Franklin Lakes, NJ) with a FACS Calibur flow cytometer (Becton Dickinson). The data was analyzed using the CellQuest software. Totally, 10,000 nucleated cells were obtained and if more than $80 \%$ of the cells were labeled, the sample was considered positive. HLAA2-positive HCC patients were denoted as the HCC group and HLA-A2-positive patients with liver cirrhosis and healthy volunteers as the non-HCC control group.

2.3. TaqMan Q-PCR. Total RNA was extracted from the tissue specimens using an RNAeasy extraction kit (Qiagen, Hilden, Germany), and cDNA was synthesized with oligonucleotide primers using a Superscript II reverse transcriptase Kit (Promega, Madison, WI) in accordance with the manufacturer's instructions. The $M A G E-A 3$ gene primers and probes were designed using ABI PRISM Primer Express 3 (Applied Biosystems, Foster City, CA). Fluorescent dye, FAM, was used as a reporter signal and $G A P D H$ was used as an internal control. $M A G E-A 3$ : forward primer: $5^{\prime}$-GGAGCA CTGAAGGAGAAGATCTG-3' ${ }^{\prime}$, reverse primer: $5^{\prime}$-TGACTC TGGTCAGGGCAACA-3', probe: $5^{\prime}$-FAM-TGGGTCTCC ATTGCCCAGCTCC-TAMRA-3'; GAPDH: forward primer: 5'-GAAGGTGAAGGTCGGAGTC-3', reverse primer: 5'GAAGATGGTGATGGGATTTC- $3^{\prime}$, probe: $5^{\prime}$-FAM-CAA GCTTCCCGTTCTCAGCC-TAMRA-3'. The PCR was run at $50^{\circ} \mathrm{C}$ for $2 \mathrm{~min}$ and $95^{\circ} \mathrm{C}$ for $10 \mathrm{~min}$ followed by 40 cycles of $92^{\circ} \mathrm{C}$ for $15 \mathrm{sec}$ and $60^{\circ} \mathrm{C}$ for $1 \mathrm{~min}$. All the reactions were performed at least in duplicate and three times independently. The PCR products were resolved by agarose gel electrophoresis and analyzed using the ABI Prism7000 detection system (Applied Biosystems). A $\mathrm{C}_{T}$ (threshold cycle) value less than 30 was considered positive.

2.4. Detection of MAGE-A3-Specific CTLs in Peripheral Blood. Ten $\mathrm{mL}$ peripheral venous blood was obtained from the study subjects who were HLA-A2-positive. After heparin anticoagulation, peripheral blood mononuclear cells (PBMCs) were isolated by density gradient centrifugation and $1 \times 10^{7} \mathrm{PBMCs}$ were incubated with anti-CD8 MicroBeads (MiltenyiBiotec, BergischGladbach, Germany) at $4^{\circ} \mathrm{C}$ for $30 \mathrm{~min}$. Approximately $1 \times 10^{6} \mathrm{CD}^{+} \mathrm{T}$ lymphocytes were isolated using MS Columns (MiltenyiBiotec). The five MHC-dimer (pentamer) MAGE-A3 epitope (p112-120, KVAELVHFL) complexes containing polypeptide were marked with phycoerythrin (PE), thus enabling recognition and binding to $\mathrm{T}$ cell receptors of $\mathrm{CD}^{+} \mathrm{T}$ cells against the antigen peptide. Pentamer staining was performed as previously described [20, 21]. FACSCalibur flow cytometry was used for detection, and data were acquired and analyzed using the CellQuest software. The lymphocyte gate was set to count $20,000 \mathrm{CD}^{+} \mathrm{T}$ cells and 20,000 double-positive CD8-FITC ${ }^{+}$pentamer- $\mathrm{PE}^{+}$cells (MAGE-A3-specific $\mathrm{CD}^{+} \mathrm{T}$ cells) and frequency was expressed by the percentage of double-positive cells.
2.5. Detection of Serum AFP Levels. Serum AFP levels were detected by electrochemiluminescence using Roche E170 instruments and commercial kits (Roche, Basel, Switzerland). AFP > $200 \mathrm{ng} / \mathrm{mL}$ was considered positive for HCC.

2.6. Follow Up. The follow-up was carried out once every 3 or 4 months for the first 3 years and then 6 months afterwards and consisted of physical examination, routine blood chemistry, serum AFP, chest X-ray, and abdominal ultrasound. In cases with suspicious lesions upon ultrasound or elevated AFP, further examinations (contrast-enhanced ultrasound, CT or MRI) were conducted to confirm or to rule out recurrence. Survival was defined as the time between the date of surgery to death of any cause.

2.7. Statistical Analysis. All data were expressed as mean \pm standard deviation (SD) and analyzed using the SPSS 19.0 (SPSS Inc., Chicago, IL) and GraphPad Prism5.0. Correlation between the frequency of MAGE-A3 peptide-specific CTLs and MAGE-A3 mRNA expression in HLA-A2-positive HCC patients was examined by nonparametric Pearson correlation analysis. Differences in the frequency of MAGE-A3-specific $\mathrm{CD}^{+} \mathrm{T}$ cells in peripheral blood among the three groups were compared by one-way analysis of variance. Student's $t$ test was used to analyze differences between the HCC group and non-HCC group. The receiver operating characteristic (ROC) curve and area under the curve (AUC) were used to delineate serum AFP or MAGE-A3-specific CTLs frequency as the efficiency index of diagnosis of HCC. The best possible cut off points were defined at the highest Youden index $(J=\operatorname{Sen}+$ Spe -1$)$. For combined detection of MAGE-A3specific CTL frequency and serum AFP, logistic regression analysis was carried out before ROC curve analysis. To study the influence of MAGE-A3-specific CTL prevalence on prognosis, we applied the Kaplan-Meier method and the logrank test. $P$ values less than 0.05 were considered statistically significant.

\section{Results}

3.1. HCC Patients Exhibit Elevated Frequency of MAGE-A3Specific CTLs. Our HLA-A2 antigen phenotyping analysis by flow cytometry revealed 85 (48.6\%) HLA-A2-positive cases from 175 HCC patients, 38 cases (47.5\%) from 80 liver cirrhosis patients, and 50 cases (47.6\%) from 105 healthy volunteers. We examined the frequency of $\mathrm{CD}^{+}{ }^{+}$-specific CTLs binding to MAGE-A3 epitopes by pentamer staining and flow cytometry. We found that the frequency of MAGEA3 binding CTLs in the peripheral blood of 85 HLA-A2positive $\mathrm{HCC}$ cases was $0.71 \pm 0.25 \%$, which was significantly higher than that of cirrhotic patients $(0.27 \pm 0.14 \%, P<$ 0.001) (Figure 1(a)). Few or no $\mathrm{CD}^{+} / \mathrm{MAGE}-\mathrm{A} 3$ doublepositive cells were detected in the healthy subjects $(0.20 \pm$ $0.10 \%)$. Furthermore, there was a marked difference in the frequency of $\mathrm{CD}^{+}{ }^{+}$-specific CTLs binding to MAGE-A3 epitopes between the HCC group and the non-HCC group $(P=$ 0.0021) (Figure 1(b)). 

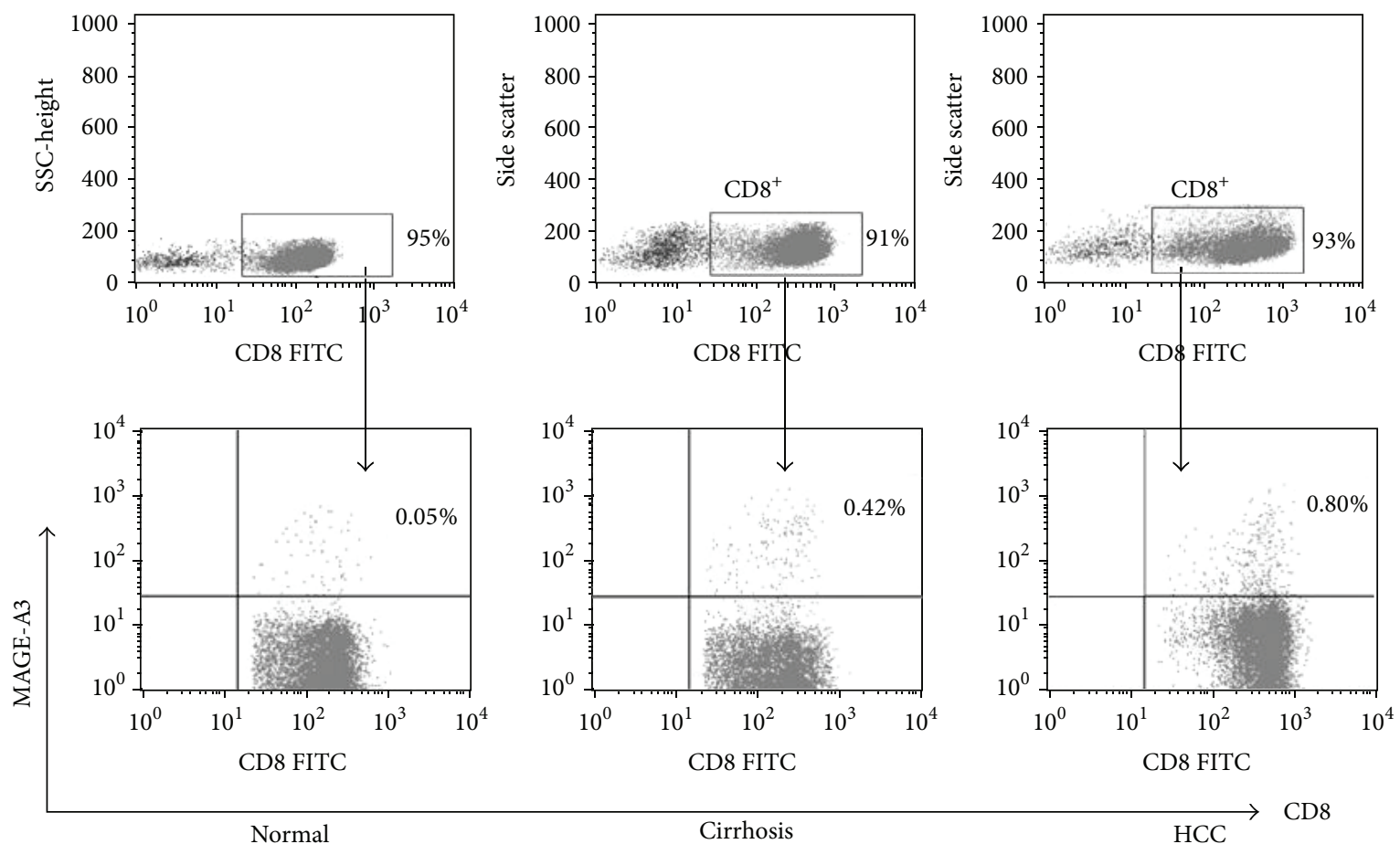

(a)
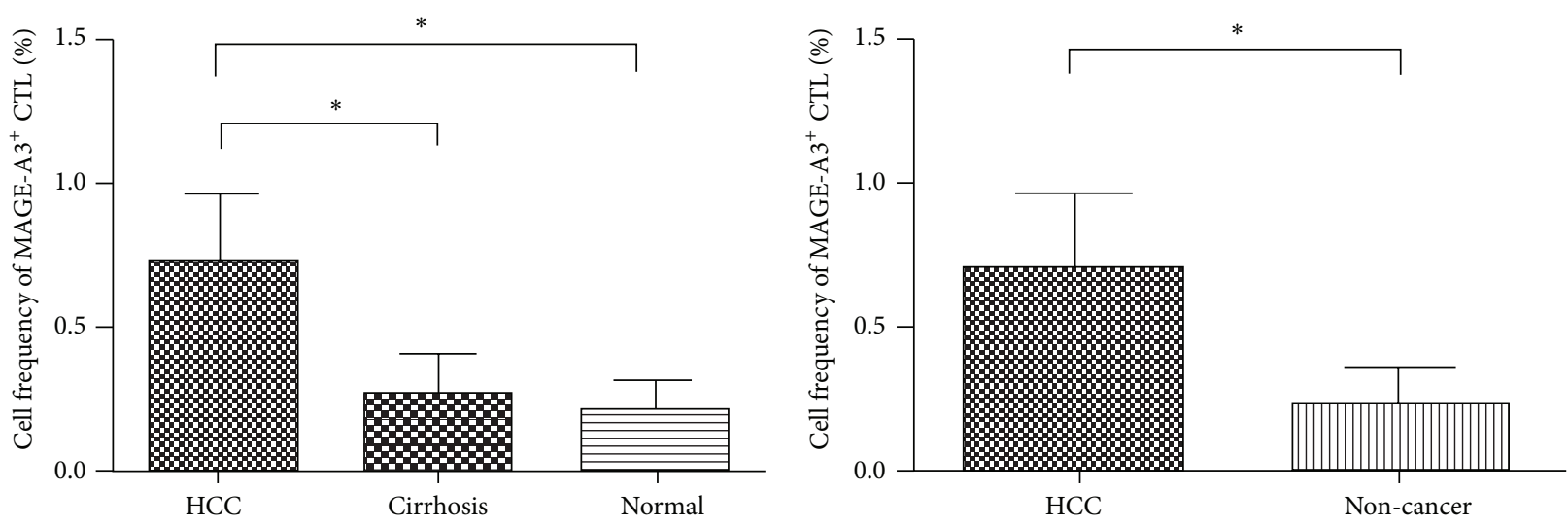

(b)

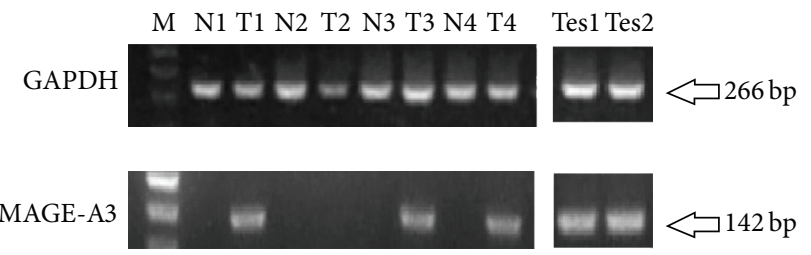

(c)

Figure 1: (a) Frequency of MAGE-A3-specific cytotoxic T lymphocytes (CTLs). The percentages of CD8 ${ }^{+} \mathrm{MAGE}^{-\mathrm{A}} 3^{+} \mathrm{CTL}$ in $\mathrm{CD} 8^{+} \mathrm{T}^{-c e l l}$ populations from HCC patients $(n=85)$, liver cirrhosis patients $(n=38)$, and healthy controls $(n=50)$. The results shown are representative of at least three independent experiments; $P<0.001$. (b) Histograms of the frequency of MAGE-A3 ${ }^{+}$CTLs in the HCC group, liver cirrhosis group, and healthy controls. Box bars show the differences in frequency among the three groups (one-way ANOVA, $P<0.001$ ). The data are presented in two groups: the cancer group, and non-cancer control group (independent $t$-test, $P<0.001$ ). ${ }^{*} P<0.05$. (c) MAGE-A3 mRNA expression in hepatocellular carcinoma (HCC) and adjacent non-cancerous tissues and testis tissues. GAPDH was used as internal control. M: 100 bp ladder marker; T: tumor; N: adjacent tissue; Tes: testis tissue. 
TABLE 2: Diagnostic performance of frequency of MAGE-A3-specific CTLs and serum AFP singly or in combination for HCC stratified by TNM and clinical stages.

\begin{tabular}{|c|c|c|c|c|c|c|c|c|}
\hline TNM stage & T1N0M0 & T2N0M0 & T3aN0M0 & T3bN0M0 & T4N0M0 & AnyTN1M0 & AnyTanyNM1 & Total cases \\
\hline Clinical stage & I & II & IIIA & IIIB & IIIC & IVA & IVB & \\
\hline HCC, $N(\%)$ & $50(58.8)$ & $15(17.7)$ & $10(11.8)$ & $3(3.5)$ & $1(1.2)$ & $3(3.5)$ & $3(3.5)$ & $85(100)$ \\
\hline MAGE-A3 ${ }^{+}$CTL $\geq 0.67 \%$ & $36(72.0 \%)$ & $11(73.3 \%)$ & $4(40.0 \%)$ & $1(33.3 \%)$ & $0(0 \%)$ & $1(33.3 \%)$ & $1(33.3 \%)$ & $54(63.5 \%)$ \\
\hline $\mathrm{AFP} \geq 200 \mathrm{ng} / \mathrm{mL}$ & $15(30.0 \%)$ & $4(26.7 \%)$ & $4(40.0 \%)$ & $2(66.7 \%)$ & $0(0 \%)$ & $1(33.3 \%)$ & $1(33.3 \%)$ & $27(31.8 \%)$ \\
\hline $\begin{array}{l}\text { MAGE-A3 } 3^{+} \mathrm{CTL} \geq 0.67 \% \\
\text { or } \mathrm{AFP} \geq 200 \mathrm{ng} / \mathrm{mL}\end{array}$ & $42(84.0 \%)$ & $12(80.0 \%)$ & $5(50.0 \%)$ & $2(66.7 \%)$ & $0(0 \%)$ & $2(66.7 \%)$ & $2(66.7 \%)$ & $65(76.5 \%)$ \\
\hline
\end{tabular}

We further determined MAGE-A3 mRNA expression by qPCR. We found that 54.6\% (24/44) of the HLA-A2-positive tumor tissue specimens expressed MAGE-A3 mRNA, which, however, was not detectable in adjacent normal tissues (Figure 1(c)). Pearson correlation analysis revealed no correlation between MAGE-A3 mRNA expression in HCC tissues and the frequency of MAGE-A3-positive CTLs in HCC patients (Pearson correlation coefficient $=0.131, P>0.05$ ).

3.2. Diagnostic Performance of Serum AFP and Frequency of MAGE-A3-Specific CTLs for HCC. At a cutoff value of $200 \mathrm{ng} / \mathrm{mL}$ for AFP, $27(31.8 \%, 27 / 85)$ cases were diagnosed with HCC. At a cutoff value of $\geq 0.67 \%$ for the frequencies of MAGE-A3-epitope-specific CTLs, 63.5\% (54/85) of the HLA-A2-positive HCC patients were diagnosed with HCC (Table 2). When the two parameters were combined, $76.5 \%$ $(65 / 85)$ of the patients were diagnosed with HCC. For early stage HCC patients (TNM I and II), 83.1\% (54/65) were diagnosed by the combined use of the two parameters whereas only $29.2 \%(19 / 65)$ early stage patients were diagnosed using AFP alone and $72.3 \%$ (47/65) early stage patients were diagnosed using the frequencies of MAGE-A3-epitopespecific CTLs alone.

ROC curve analysis showed that the AUC of serum AFP was 0.613 (Figure 2(a)). At a cutoff value of $200 \mathrm{ng} / \mathrm{mL}$, the sensitivity of serum AFP for diagnosing HCC was $31.8 \%$, and the specificity was $98.9 \%$ with the Youden index at 0.307 . For the frequency of MAGE-A3-positive CTLs, the AUC was 0.873 (Figure 2(b)). At a cutoff value of $0.67 \%, 63.5 \%(54 / 85)$ of HCC patients exhibited a specific MAGE-A3 CTL response to HLA-A2-restricted epitopes. In contrast, 38 (100\%) patients with liver cirrhosis and $50(100 \%)$ healthy volunteers were negative for this response. The diagnostic sensitivity of the frequency of MAGE-A3-positive CTLs at $0.67 \%$ was $68.6 \%$ and the specificity was $100 \%$ with the Youden index at 0.686 .

While serum AFP did not correlate with the frequency of MAGE-A3-specific CTLs (Pearson correlation coefficient $=0.093, P=0.154)$, the two indicators were complementary. We analyzed the combination of the two indicators according to the decision boundary in HCC diagnosis. At a cutoff value of $200 \mathrm{ng} / \mathrm{mL}$ for AFP and at a cutoff of $0.67 \%$ for MAGEA3-specific CTLs, the ROC curve of the AUC for combined AFP and MAGE-A3-specific CTLs was 0.916 with a sensitivity of $72.9 \%$ and a specificity of $100 \%$ (Figure 2(c)). The Youden index was 0.729 . The findings indicate markedly improved diagnostic performance of combined AFP and MAGE-A3specific CTLs for diagnosing HLA-A2-positive HCC.

3.3. The Frequencies of MAGE-A3 Specific CTLs Do Not Correlate with Patient Clinicopathologic Parameters. We next analyzed the correlations between the frequency of MAGE-A3specific CTLs and patient clinicopathologic parameters such as age, sex, preoperative AFP level $(20 \mathrm{ng} / \mathrm{mL}$ and $200 \mathrm{ng} /$ $\mathrm{mL}$ ), tumor size, tumor number, HBsAg expression, ChildPugh class, TNM staging, and differentiation (Table 1). The frequency of MAGE-A3-specific CTLs exhibited no statistically significant association with any of these parameters $(P>0.05)$.

3.4. Relationship between MAGE-A3-Specific CTL Frequency and Prognosis. All 85 HCC patients were followed up for a mean duration of 34 (range, 4 to 68 ) months after surgery. Twenty-four patients died and 54 patients were alive at the time of the last follow-up. The median survival was $37 \pm 5.4$ months; the 1-year, 3-year, and 5-year survival rates were $80.8 \%, 41.3 \%$, and $27.5 \%$, respectively. The survival curve is shown in Figure 3(a). The group with MAGE-A3 specific CTL frequency above $0.67 \%$ had a median survival of $37 \pm 6.0$ months. Furthermore, the 1-year, 3-year, and 5-year survival rates were $91.3 \%, 43.0 \%$, and $21.5 \%$, respectively. In the other group with MAGE-A3 specific CTL frequency below $0.67 \%$, the median survival was $19 \pm 7.7$ months, and the 1-year, 3year, and 5-year survival rates were $63.8 \%, 45.7 \%$, and $38.1 \%$, respectively (Table 3). The Kaplan-Meier survival curves showed that patients with low cell frequency of MAGEA3 $(<0.67 \%)$ did not have a significant survival difference compared to patients with elevated cell frequency of MAGEA3 $(\geq 0.67 \%)(P=0.099)$ (Figure 3(b)).

\section{Discussion}

In China, serum AFP level is used as one of the conventional indexes of primary HCC diagnosis. However, highly and poorly differentiated HCC cells usually produce little AFP in contrast to the high levels synthesized by moderately differentiated HCC cells [22]. Therefore, the positive rate of AFP in the diagnosis of HCC is generally only $60-70 \%$ [23]. In addition, serum AFP levels in patients with liver cirrhosis can be very high, suggesting that AFP gives false positives in the diagnosis of primary HCC. 


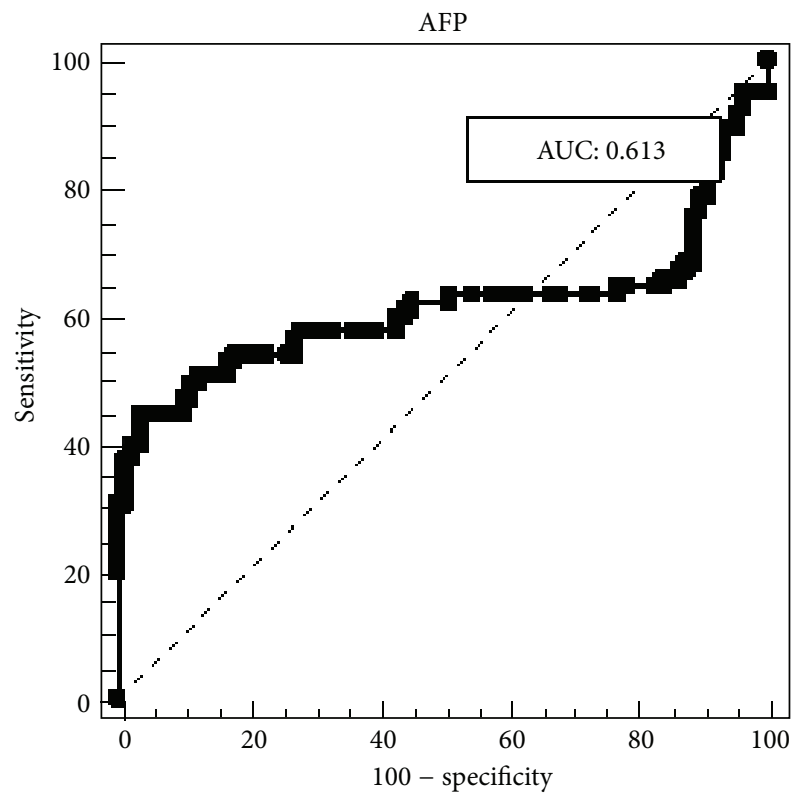

(a)

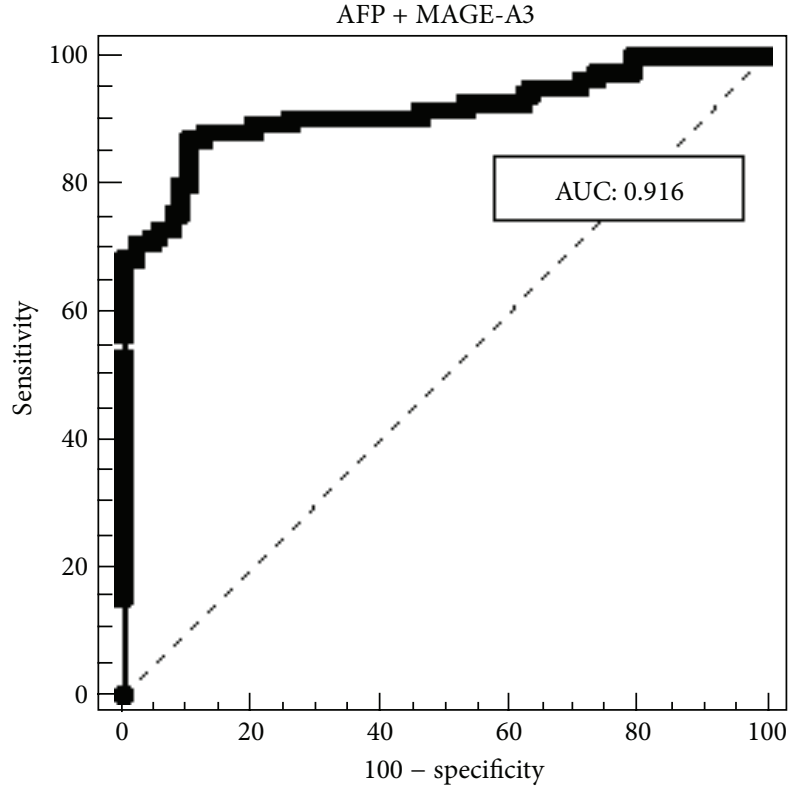

(c)

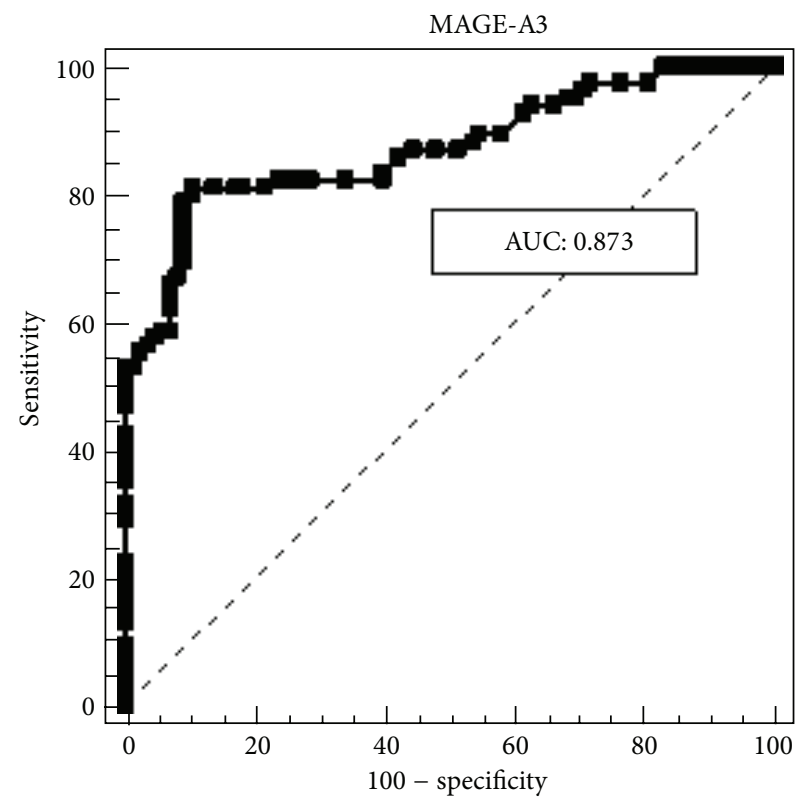

(b)

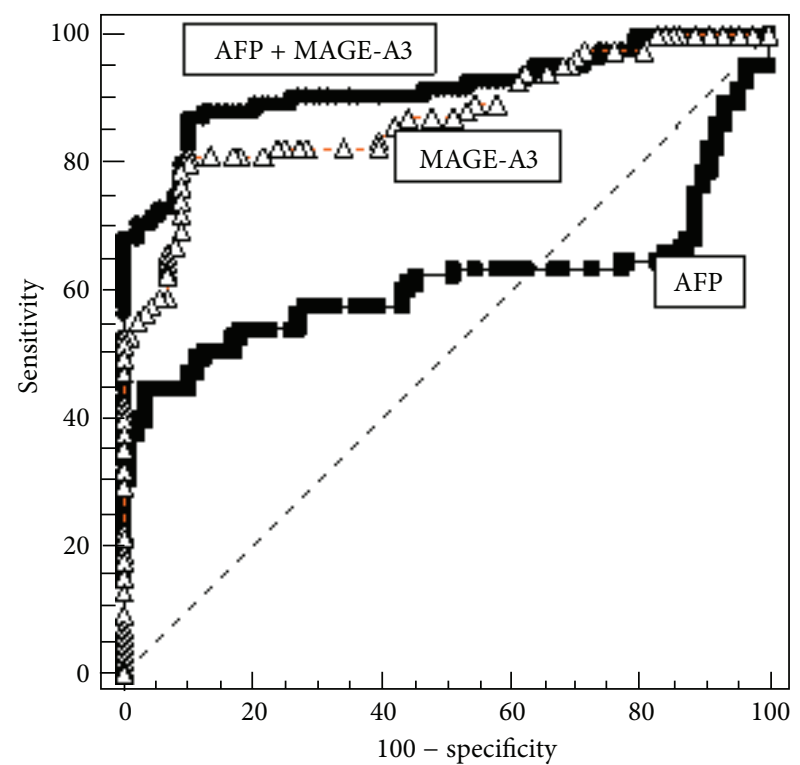

(d)

FIgURE 2: Comparison of individual or combined indicators for HCC diagnosis. ROC curves comparing AFP (a), MAGE-A3 (b), and AFP + MAGE-A3 (c) in HCC patients, liver cirrhosis patients, or healthy controls. The curves show the optimal cut-off value for the frequency of MAGE-A3 ${ }^{+}$CTLs of $0.67 \%$. AUC: area under the curve.

TABLE 3: Survival comparison of HCC groups with different frequencies of MAGE-A3 ${ }^{+}$CTL.

\begin{tabular}{|c|c|c|c|c|c|}
\hline & $\begin{array}{c}\text { Mean survival, } \\
\text { month }\end{array}$ & 1-year survival rate & 3-year survival rate & 5-year survival rate & Log rank test $P$ value \\
\hline $\begin{array}{l}\text { Cell frequency of MAGE-A3 }{ }^{+} \text {CTL } \\
\geq 0.67 \%\end{array}$ & $37 \pm 6.0$ & $91.3 \%$ & $43.0 \%$ & $21.5 \%$ & 0.099 \\
\hline $\begin{array}{l}\text { Cell frequency of MAGE-A3 }{ }^{+} \text {CTL } \\
<0.67 \%\end{array}$ & $19 \pm 7.7$ & $63.8 \%$ & $45.7 \%$ & $38.1 \%$ & \\
\hline
\end{tabular}




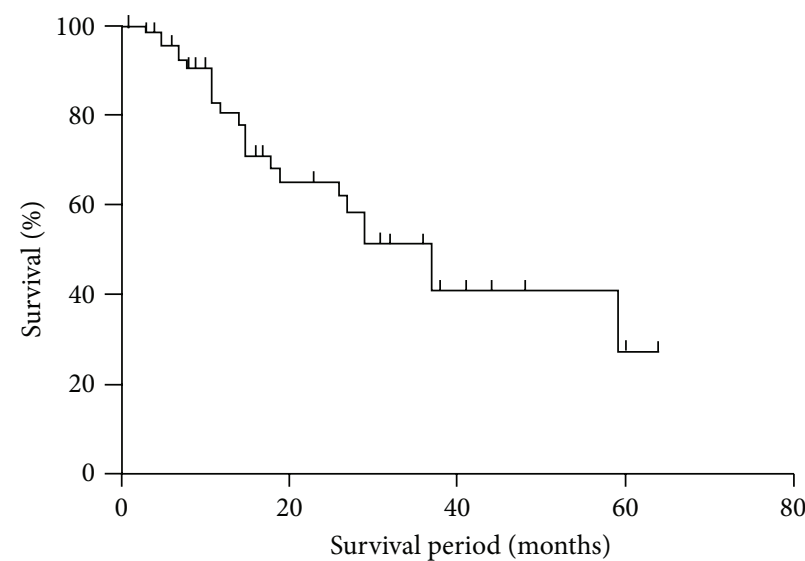

(a)

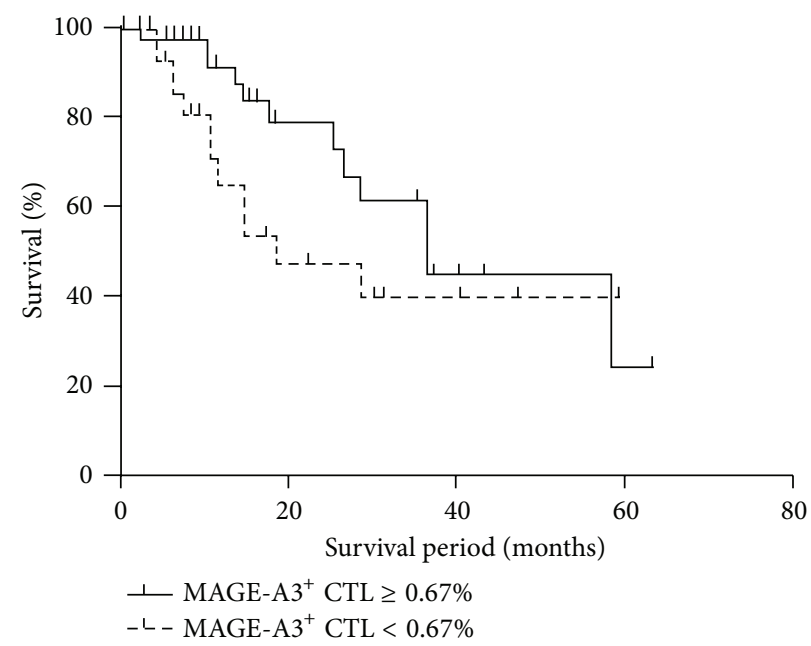

(b)

FIGURE 3: Correlation between MAGE-A3-specific CTLs and survival. (a) Overall survival curves following surgery grouped by frequency of MAGE-A3 ${ }^{+}$CTLs as calculated using the Kaplan-Meier method. (b) Statistical analysis showed that the group with MAGE-A3 ${ }^{+}$CTL frequency above $0.67 \%(n=54)$ had no significant survival difference from the other group $(n=31)(P=0.015)$.

In this study, we used pentamer staining and flow cytometry to detect the frequency of CTLs specific for HLAA2/MAGE-A3112-120 epitope in the peripheral blood of HCC patients. When the cutoff value of MAGE-A3-positive CTLs was set at $0.67 \%, 63.5 \%(54 / 85)$ patients with HCC exhibited positive results. However, a corresponding CTL response was not detected in 38 patients with liver cirrhosis and 50 healthy volunteers. Thus, the pentamer technique may be sensitive enough to detect spontaneous CTL response to a MAGEA3 specific epitope, and it may be used as an important auxiliary method to improve the diagnosis rates of HCC. We adopted the ROC curve method to evaluate the efficiency of serum AFP measurement in the diagnosis of HCC. According to the commonly used decision boundary applied in the clinical diagnosis of HCC, when the AFP-positive cutoff value was $200 \mathrm{ng} / \mathrm{mL}$, the sensitivity and specificity of AFP were $42.86 \%$ and $98.9 \%$, respectively, and the AUC was 0.613 . Combined detection of serum AFP levels and MAGE-A3specific CTLs with a cutoff of $0.67 \%$ resulted in an ROC curve with an AUC of 0.916 , which apparently improved the diagnostic sensitivity for HCC, reaching $72.9 \%$. However, the specificity remained $100 \%$ and the Youden index was 0.729 . Thus, this combined detection method clearly improved the diagnostic performance. Our results showed that using the HLA-A2/MAGE-A3 polypeptide pentamer technology, specific spontaneous CTL response to tumor-specific antigens can be quantitatively identified in the peripheral blood of patients with HCC. The MAGE-A3-specific CTL frequency and its diagnostic significance in a large cohort have not been previously investigated. In addition, we found that in the 44 HLA-A2-positive HCC cases expressing the MAGE-A3 gene, there was no correlation between MAGE-A3 mRNA expression and MAGE-A3-specific CTL response. Another study showed that many CT genes, including MAGE-A3, exhibited the phenomenon of clustering expression [24]. Various CT antigens are spontaneously expressed in tumors but in some cases, no CT antigen is expressed. Therefore, while antigen expression is not always detectable in solid tumors, CTL response to antigens may be detected in peripheral blood. For reliable assessment of specific CTL detection, qPCR data can be used as an important reference. In the future, if the diagnosis index of HCC is applied, these flexible inspection methods should be considered.

This study found that parameters including patient's gender, age, preoperative AFP level $(20 \mathrm{ng} / \mathrm{mL}$ and $200 \mathrm{ng} / \mathrm{mL})$, tumor size, tumor number, TNM staging, and differentiation had no significant correlation with the frequency of MAGE$\mathrm{A}^{+}$CTLs $(P>0.05)$. In addition, no significant correlations were observed in other studies $[25,26]$.

In addition, since the expression frequency of HLAA2 antigen reached $51.9 \%$ in Chinese Hans patients [27], we chose HLA-A2-positive patients and an HLA-A2 type pentamer. Since we only chose the HLA-A2 type pentamer, we could not cover all HCC patients in our assessment. With the progression of technology and the gradual accumulation of data, we will eventually have a variety of HLA-type pentamers at our disposal and will be able to choose antigens with stronger immunogenicity that encompass more patients.

In this study, by applying classical immunology theory and advancing technology, we analyzed the response of CTLs against a specific tumor antigen and attempted to find a diagnostic HCC "mirror" marker. This study showed that, with the relatively mature and sensitive pentamer technology, the combined detection of the frequency of CTL response to a tumor-specific antigen peptide with serum AFP levels was of value in the diagnosis of HCC, especially in cases of serum AFP-negative HCC. This novel auxiliary diagnostic mode may be established to enhance the sensitivity and specificity of classic HCC screening and improve the diagnosis rates of HCC [28]. Moreover, MAGE-A3-specific TCRs are identified 
and made ready for immune therapy [29, 30]. Further research on MAGE-A3 will enable us to apply this tumorspecific antigen CTL marker in the diagnosis of HCC.

\section{Conflict of Interests}

The authors have no conflict of interests to declare.

\section{Acknowledgment}

The work was supported by the National Natural Science Foundation of China (no. 30772119). Zhuqingqing Cui and Xin Yu are equal first authors.

\section{References}

[1] R. Siegel, D. Naishadham, and D. V. M. A. Jemal, "Cancer statistics 2013," Cancer Journal for Clinicians, vol. 63, no. 1, pp. 1130, 2013.

[2] S. Garrean, J. Hering, A. Saied, W. S. Helton, and N. J. Espat, "Radiofrequency ablation of primary and metastatic liver tumors: a critical review of the literature," American Journal of Surgery, vol. 195, no. 4, pp. 508-520, 2008.

[3] J. Dubbeld, H. Hoekstra, W. Farid et al., "Similar liver transplantation survival with selected cardiac death donors and brain death donors," British Journal of Surgery, vol. 97, no. 5, pp. 744753, 2010.

[4] Y.-L. Chen, C.-J. Ko, S.-Y. Chien et al., “Tumor size as a prognostic factor in resected small hepatocellular carcinoma: a controversy revisited," Journal of Gastroenterology and Hepatology, vol. 26, no. 5, pp. 851-857, 2011.

[5] T. M. Pawlik, K. A. Delman, J.-N. Vauthey et al., "Tumor size predicts vascular invasion and histologic grade: implications for selection of surgical treatment for hepatocellular carcinoma," Liver Transplantation, vol. 11, no. 9, pp. 1086-1092, 2005.

[6] J. Bruix, M. Sherman, J. M. Llovet et al., "Clinical management of hepatocellular carcinoma. Conclusions of the barcelona-2000 EASL conference," Journal of Hepatology, vol. 35, no. 3, pp. 421430, 2001.

[7] V. T. T. Nguyen, M. G. Law, and G. J. Dore, "Hepatitis B-related hepatocellular carcinoma: epidemiological characteristics and disease burden," Journal of Viral Hepatitis, vol. 16, no. 7, pp. 453463, 2009.

[8] H. B. El-Serag, "Hepatocellular carcinoma: recent trends in the United States," Gastroenterology, vol. 127, pp. S27-S34, 2004.

[9] Z. Y. Tang, Y. Q. Yu, X. D. Zhou, Z. C. Ma, and Z. Q. Wu, "Progress and prospects in hepatocellular carcinoma surgery," Annales de Chirurgie, vol. 52, pp. 558-563, 1998.

[10] Z. Y. Tang, "Hepatocellular carcinoma: cause, treatment and metastasis," World Journal of Gastroenterology, vol. 7, no. 4, pp. 445-454, 2001.

[11] R. Kuromatsu, M. Tanaka, and K. Tanikawa, "Serum alpha-fetoprotein and lens culinaris agglutinin-reactive fraction of alphafetoprotein in patients with hepatocellular carcinoma," Liver, vol. 13, no. 4, pp. 177-182, 1993.

[12] A. J. G. Simpson, O. L. Caballero, A. Jungbluth, Y.-T. Chen, and L. J. Old, "Cancer/testis antigens, gametogenesis and cancer," Nature Reviews Cancer, vol. 5, no. 8, pp. 615-625, 2005.

[13] J.-R. Peng, H.-S. Chen, D.-C. Mou et al., "Expression of cancer/testis (CT) antigens in Chinese hepatocellular carcinoma and its correlation with clinical parameters," Cancer Letters, vol. 219, no. 2, pp. 223-232, 2005.

[14] Y. Wang, K.-J. Han, X.-W. Pang et al., "Large scale identification of human hepatocellular carcinoma-associated antigens by autoantibodies," Journal of Immunology, vol. 169, no. 2, pp. 11021109, 2002.

[15] X. A. Yang, X. Y. Dong, H. Qiao et al., "Immunohistochemical analysis of the expression of FATE/BJ-HCC-2 antigen in normal and malignant tissues," Laboratory Investigation, vol. 85, no. 2, pp. 205-213, 2005.

[16] N. Miyagawa, K. Kono, K. Mimura, H. Omata, H. Sugai, and H. Fujii, "A newly identified MAGE-3-derived, HLA-A24restricted peptide is naturally processed and presented as a CTL epitope on MAGE-3-expressing gastrointestinal cancer cells," Oncology, vol. 70, no. 1, pp. 54-62, 2006.

[17] Y. Zhang, N. Renkvist, Z. Sun et al., "A polyclonal anti-vaccine CD4 $\mathrm{T}$ cell response detected with HLA-DP4 multimers in a melanoma patient vaccinated with MAGE-3.DP4-peptidepulsed dendritic cells," European Journal of Immunology, vol. 35, no. 4, pp. 1066-1075, 2005.

[18] Y. Zhang, Z. Sun, H. Nicolay et al., "Monitoring of anti-vaccine CD4 $\mathrm{T}$ cell frequencies in melanoma patients vaccinated with a MAGE-3 protein," Journal of Immunology, vol. 174, no. 4, pp. 2404-2411, 2005.

[19] D. Atanackovic, N. K. Altorki, E. Stockert et al., "Vaccineinduced CD4+ $\mathrm{T}$ cell responses to MAGE-3 protein in lung cancer patients," Journal of Immunology, vol. 172, no. 5, pp. 3289-3296, 2004.

[20] C. J. Cohen, Y. Zhao, Z. Zheng, S. A. Rosenberg, and R. A. Morgan, "Enhanced antitumor activity of murine-human hybrid Tcell receptor (TCR) in human lymphocytes is associated with improved pairing and TCR/CD3 stability," Cancer Research, vol. 66, no. 17, pp. 8878-8886, 2006.

[21] L. D. Anderson Jr., D. R. Cook, T. N. Yamamoto, C. Berger, D. G. Maloney, and S. R. Riddell, "Identification of MAGE-C1 (CT-7) epitopes for T-cell therapy of multiple myeloma," Cancer Immunology, Immunotherapy, vol. 60, no. 7, pp. 985-997, 2011.

[22] H. Toyoda, T. Kumada, T. Tada et al., "Clinical utility of highly sensitive Lens culinaris agglutinin-reactive alpha-fetoprotein in hepatocellular carcinoma patients with alpha-fetoprotein <20 ng/mL," Cancer Science, vol. 102, no. 5, pp. 1025-1031, 2011.

[23] F. Trevisani, P. E. D’Intino, A. M. Morselli-Labate et al., "Serum $\alpha$-fetoprotein for diagnosis of hepatocellular carcinoma in patients with chronic liver disease: influence of HBsAg and antiHCV status," Journal of Hepatology, vol. 34, no. 4, pp. 570-575, 2001.

[24] M. J. Scanlan, A. O. Gure, A. A. Jungbluth, L. J. Old, and Y.-T. Chen, "Cancer/testis antigens: an expanding family of targets for cancer immunotherapy," Immunological Reviews, vol. 188, pp. 22-32, 2002.

[25] M. A. Kienstra, H. B. Neel, S. E. Strome, and P. Roche, "Identification of NY-ESO-1, MAGE-1, and MAGE-3 in head and neck squamous cell carcinoma," Head and Neck, vol. 25, no. 6, pp. 457-463, 2003.

[26] M.-S. Park, J.-W. Park, C.-H. Jeon, K.-D. Lee, and H.-K. Chang, "Expression of melanoma antigen-encoding genes (MAGE) by common primers for MAGE-A1 to -A6 in colorectal carcinomas among Koreans," Journal of Korean medical science, vol. 17, no. 4, pp. 497-501, 2002.

[27] L.-H. Cheng, S.-Z. Jin, S.-Q. Gao et al., "Difference in HLA$A * 02$ allele distribution between Han populations in South and 
North China," Di Yi Jun Yi Da Xue Xue Bao, vol. 25, no. 3, pp. 321-324, 2005.

[28] J. A. Marrero, "Screening tests for hepatocellular carcinoma," Clinics in Liver Disease, vol. 9, no. 2, pp. 235-251, 2005.

[29] T. Straetemans, M. Van Brakel, S. Van Steenbergen et al., “TCR gene transfer: MAGE-C2/HLA-A2 and MAGE-A3/HLA-DP4 epitopes as melanoma-specific immune targets," Clinical and Developmental Immunology, vol. 2012, Article ID 586314, 14 pages, 2012.

[30] N. Chinnasamy, J. A. Wargo, Z. Yu et al., "A TCR targeting the HLA-A $* 0201$-restricted epitope of MAGE-A3 recognizes multiple epitopes of the MAGE-A antigen superfamily in several types of cancer," Journal of Immunology, vol. 186, no. 2, pp. 685-696, 2011. 


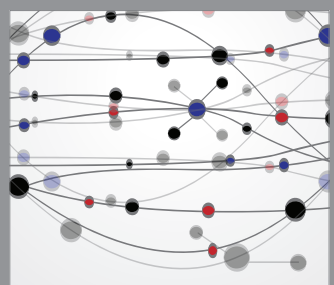

The Scientific World Journal
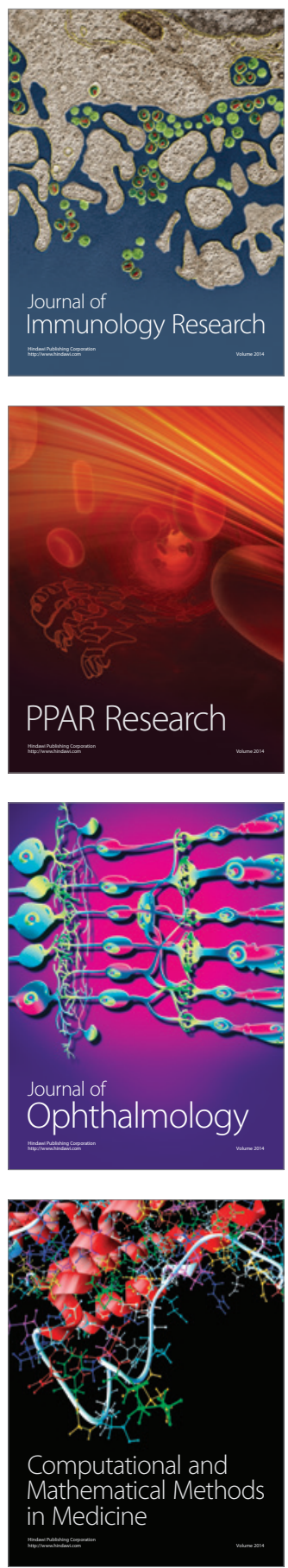

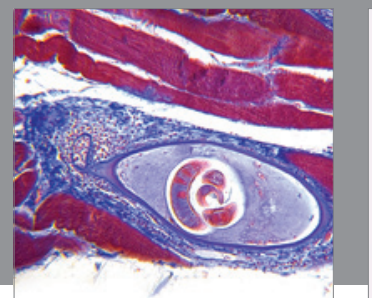

Gastroenterology

Research and Practice
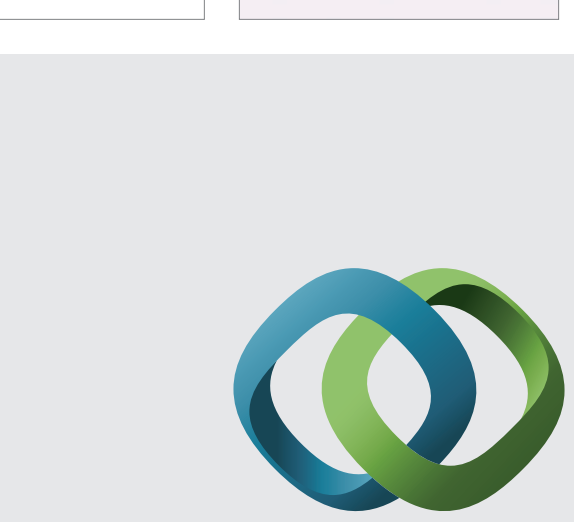

\section{Hindawi}

Submit your manuscripts at

http://www.hindawi.com
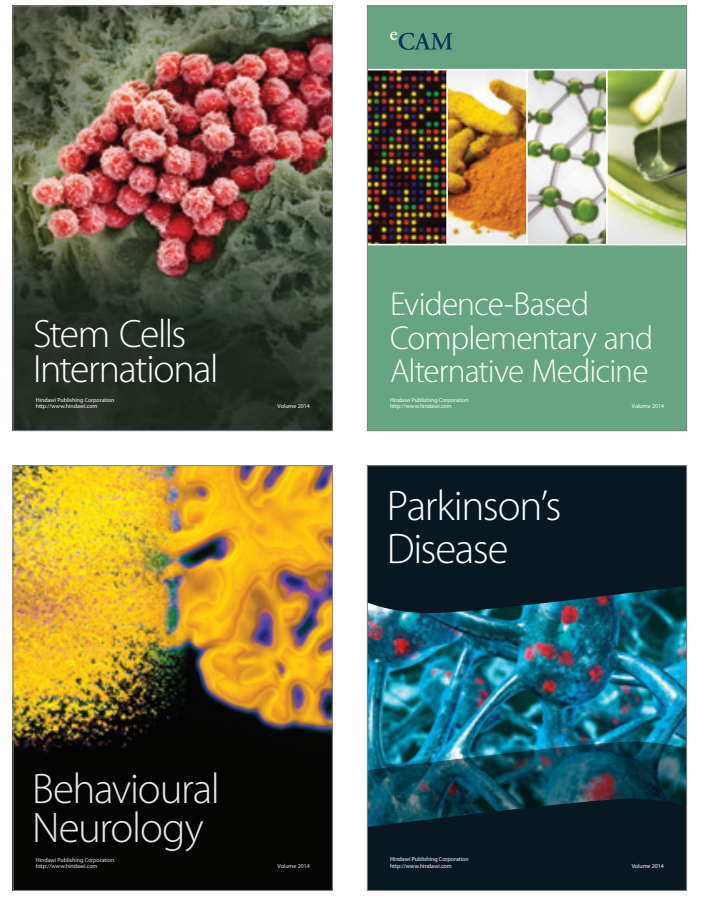
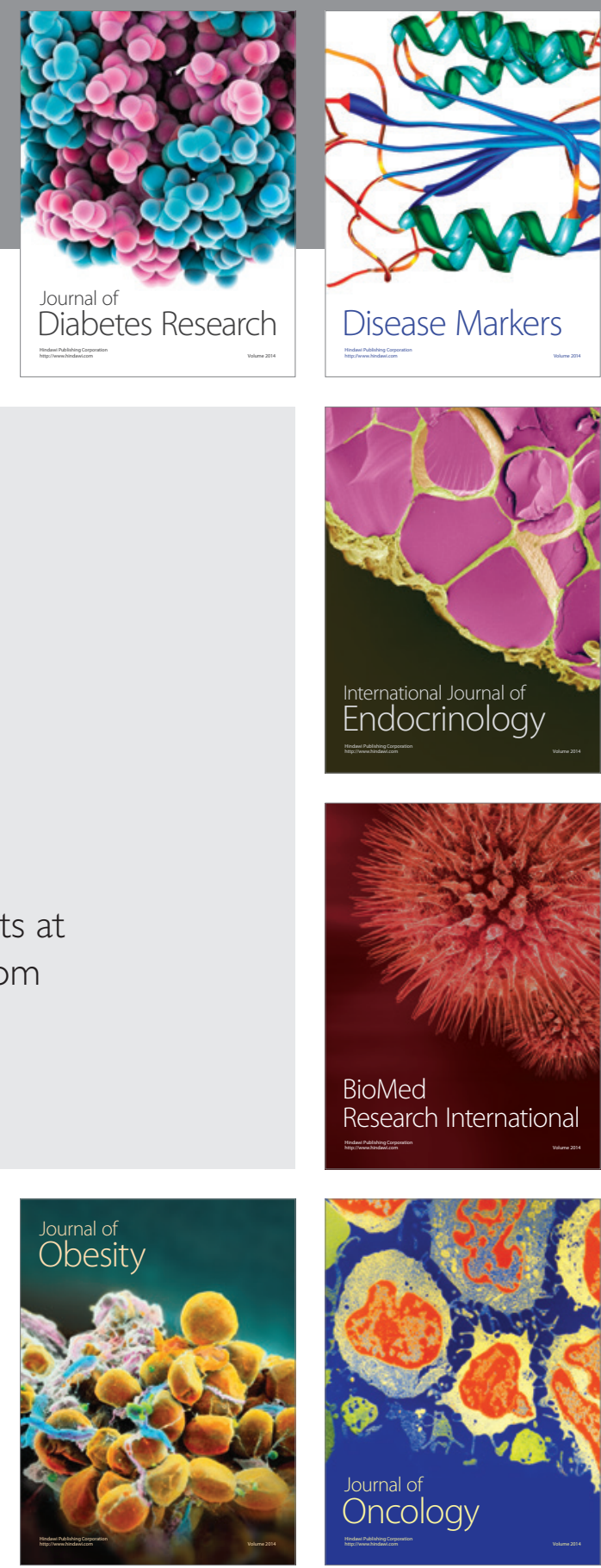

Disease Markers
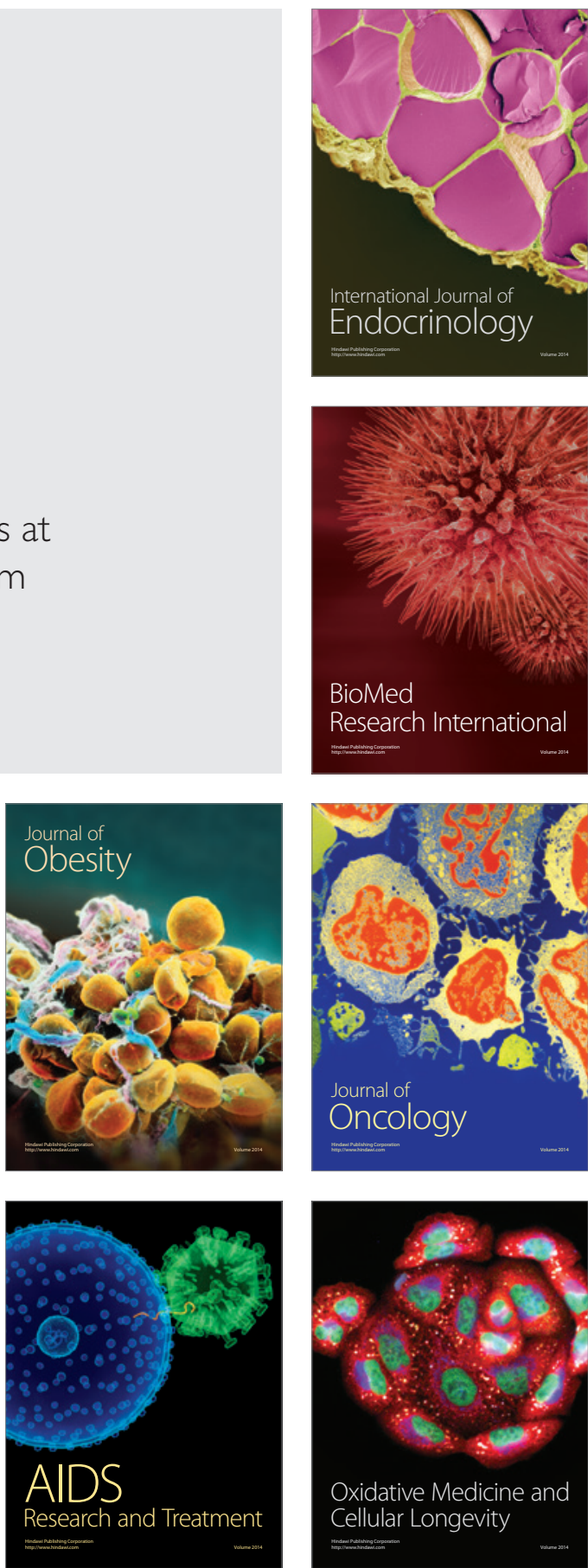